\section{ALTERNATIVE TREATMENTS FOR MENISCAL INJURIES}

\author{
R. VERDONK
}

From Ghent University Hospital, Belgium

Historical review. "By arterial injection with an opaque medium, one can discern a network of fine vessels from the capsule, entering the convex border of the meniscus but disappearing almost immediately. Because of this, one might expect healing in peripheral meniscus detachments, but none in tears limited to the semilunar cartilage itself".

These were the opening sentences of King's paper at a meeting of the American Academy of Orthopaedic Surgeons in St Louis, Missouri on 13 January 1936. He performed several experiments to assess the healing capacity of the internal semilunar cartilage of the knee in dogs. Incisions made in and around the semilunar cartilages seemed to heal to a varying degree in relation to contact with the synovium on the outer edge of the meniscus.

These findings indicated that (Fig. 1): 1) tears confined to the semilunar cartilage probably never heal; 2) a torn meniscus may heal by connective tissue if the tear communicates with the synovial membrane; 3) a complete transverse or oblique tear results in some separation of the fragments and within three weeks the intervening space fills with firm connective tissue arising from the synovium, indicating the time necessary for complete fixation; and 4) a meniscus partially torn from its peripheral attachment heals readily in a normal anatomical position.

Why save the meniscus? On 16 November 1883 Thomas Annandale was the first to suture a medial meniscus, but arthrotomy and meniscectomy have since become common orthopaedic procedures. In the 1950s and the 1960s total meniscectomy was performed for almost any meniscal tear suspected on clinical examination. In the last two decades, arthroscopy has allowed adequate meniscectomy, following the technical rules laid down by authors such as Jackson.

Between 1970 and 1980 it was shown that a carefully

R. Verdonk, Professor of Orthopaedic Surgery

Ghent University Hospital, De Pintelaan 185, 9000 Ghent, Belgium.

Updated text and illustrations, printed with the permission of EFORT. The original article appears in European Instructional Course Lectures Vol. 3, 1997.
INSTRUCTIONAL COURSE LECTURE executed arthroscopic meniscectomy for a torn medial meniscus provided full functional restoration in more than $90 \%$ of cases, with short-term results comparable with those of open meniscectomy. In the longer term, factors such as varus malalignment and mechanical overload increase the risk of degeneration of load-bearing cartilage (Fig. 2). The buffer function of the semilunar cartilage between the femoral condyle and medial tibial plateau is lost, and the stabilising factor, the meniscal wall, is also lacking. The result is an increased anteroposterior shift of the femoral condyle in relation to the medial tibial plateau (Fig. 3). Any ligamentous laxity produced by the initial trauma will increase the degenerative changes in the loadbearing area. Even more important, but less controllable, is the magnitude of the mechanical load. This will depend on the weight of the patient and on work- and sports-related activity.

The same factors also apply to older patients. The shortterm results of accurate arthroscopic meniscectomy are better than those of open total meniscectomy because of the preservation of the meniscal wall. Again, the quality of the load-bearing cartilage will determine the functional out-
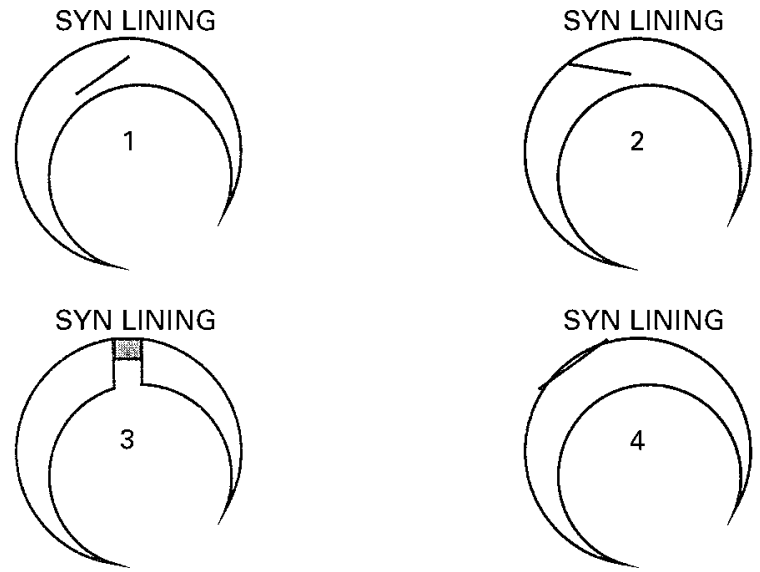

Fig. 1

Diagram to show a tear in the meniscus which probably never heals (1), a tear which probably heals by connective tissue since it communicates with the synovial membrane (2), a complete transverse or oblique tear which heals by connective tissue arising from the synovial membrane (3), and a partial tear of the meniscus from its peripheral attachment which will readily heal in its anatomical position (4). 


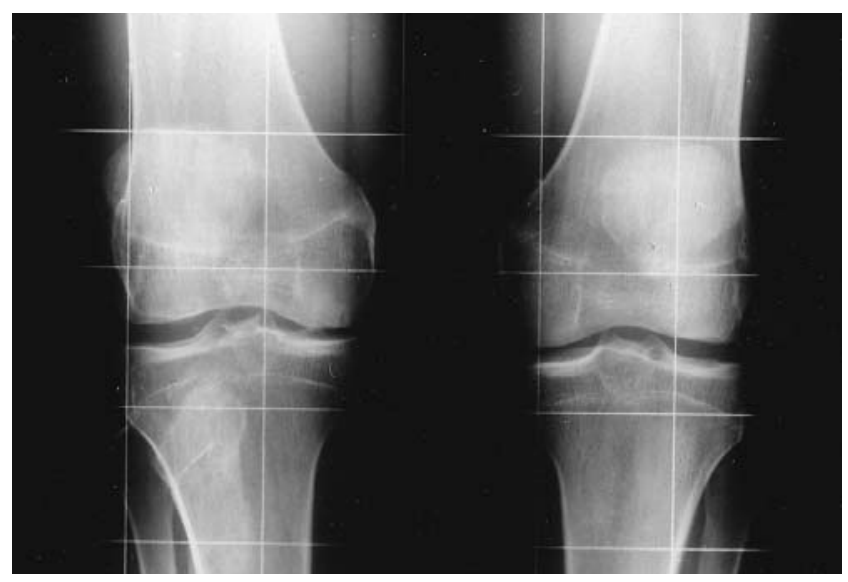

Fig. 2

After medial meniscectomy, factors such as varus malalignment and mechanical overload increase the risk of degeneration in the load-bearing medial compartment.
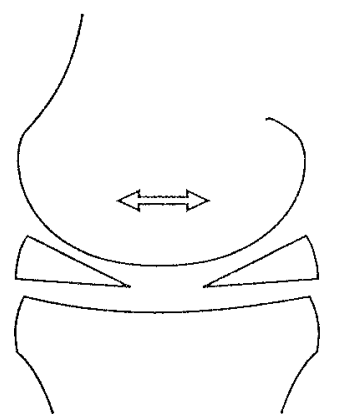

Non-removed meniscus Normal AP shift

\section{Fig. 3}
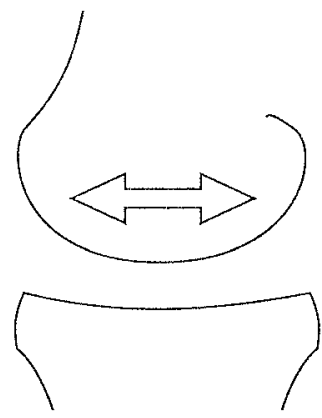

Removed meniscus Increased AP shift

After total meniscectomy an increased anteroposterior shift can be expected.

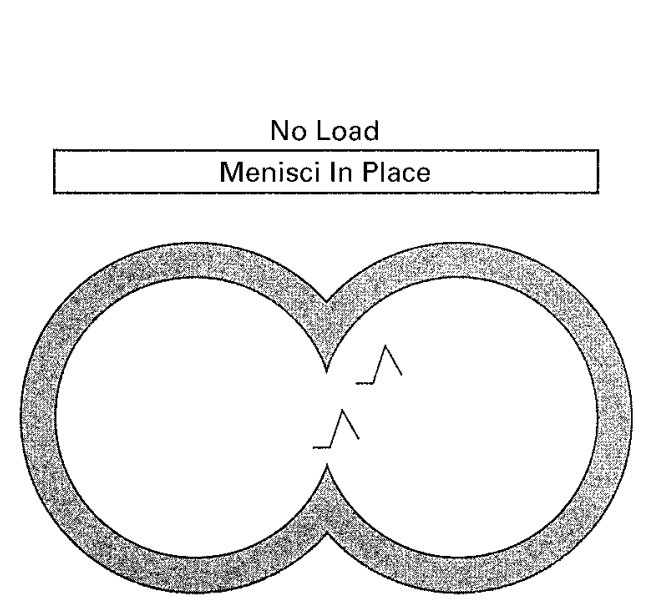

Fig. 4

In vitro measurement of the load-bearing function of the menisci shows that in non-load conditions the contact areas are primarily on the menisci. About $10 \%$ of the cartilage is in contact in non-load conditions, near the medial tibial spine. come in this age group and, in the long term, only $50 \%$ of the patients will continue to benefit.

These poor to fair results in older patients draw attention to the negative consequences of meniscectomy and suggest that meniscal suture should be performed whenever this is feasible. A functionally competent knee and an anatomical restoration can be expected in $90 \%$ of cases. ${ }^{2}$

If chondral congruity is indeed improved by the presence of the medial meniscus under loading conditions, then this also applies to the lateral compartment. The convex lateral femoral condyle articulates with an almost convex lateral tibial plateau. The contact area between the joint surfaces is flattened and widened only because of the presence of the $\mathrm{O}$-shaped lateral meniscus. More caution is necessary in the treatment of a lateral meniscal lesion than of a torn medial meniscus. Clinical experience has shown that problems may arise even after the correct and adequate resection of a torn lateral meniscus.

\section{MENISCAL FUNCTION}

Load-bearing function. Since degenerative changes are often observed after meniscectomy ${ }^{3,4}$ it has been suggested that the menisci have an important role in load transmission across the knee. ${ }^{5}$ In non-loaded conditions the contact is primarily on the menisci (Fig. 4). Load-bearing areas in the normal knee have been measured. Only $10 \%$ of the cartilage is in contact at no load, and this is restricted to an area close to the medial tibial spine (Fig. 4). Under load, contact occurs both on the menisci and on the exposed articular cartilage. It was shown by Ahmed and Burke ${ }^{6}$ that $50 \%$ of the compressive load was transmitted through the menisci in extension (Fig. 5) and $85 \%$ at $90^{\circ}$ of flexion (Fig. 6). These findings confirm that the menisci transmit a sig-

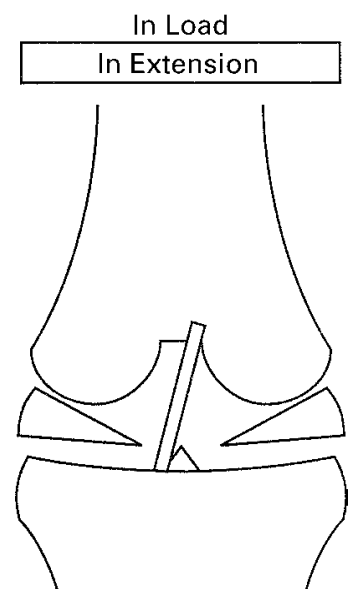

Fig. 5

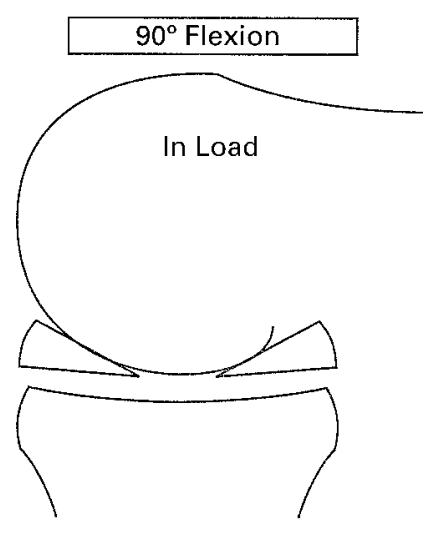

Fig. 6
Figure 5 - In vitro measurement of the load-bearing function of the menisci shows that $50 \%$ of the compressive load is transmitted through the menisci in extension. Figure 6 - In vitro measurement of the load-bearing function of the menisci shows that $85 \%$ of the compressive load is transmitted through the menisci at $90^{\circ}$ of flexion. 


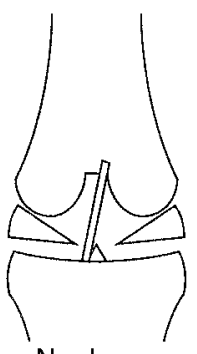

No. knee

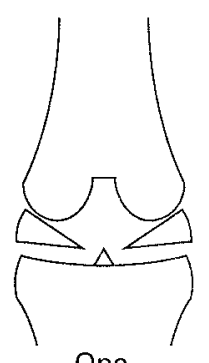

One
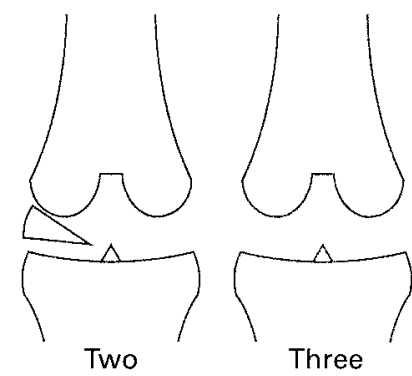

Fig. 7
'One-two-three' knee: one stands for rupture of the anterior cruciate ligament, two for subsequent medial meniscectomy, and three for final lateral meniscectomy and severe incapacity.

nificant part of the load from the femur to the tibia.

Shock absorption. Because of their visco-elasticity, the menisci attenuate shock waves generated by impulse loading in normal gait.

Maintenance of joint stability. There is no agreement about the stabilising role of the menisci. Increased laxity has been reported after meniscectomy ${ }^{7}$ but other studies show no such increase. 8 Meniscectomy in a ligamentdeficient knee, especially with lack of the anterior cruciate ligament, significantly increases joint laxity. ${ }^{9}$ Degenerative evolution after such combined lesions has been observed, and has been referred to as the 'one-two-three knee' (Fig. 7). 'One' stands for solitary rupture of the anterior cruciate ligament (ACL), 'two' for subsequent medial meniscectomy and 'three' for final lateral meniscectomy, which ultimately leads to severe incapacity.

Joint lubrication. Two of the several forms of lubrication should be considered: boundary lubrication and fluid film lubricant. Lubricin appears to function as a boundary lubricant in synovial joints. There are two different forms of fluid film lubrication: a hydrodynamic mechanism in which load-bearing surfaces work tangentially on each other, and a squeeze-film mechanism in which load-bearing surfaces move perpendicularly towards each other. The actual knee lubrication mechanism is elastohydrodynamic in nature. ${ }^{7}$

\section{MENISCAL REPAIR}

Except for cases with associated lesions for which an open arthrotomy is required, meniscectomy should always be performed arthroscopically. This improves the efficiency of the procedure. Nevertheless, meniscal repair should always be considered during evaluation. Important factors are the clinical signs of associated lesions, and the type, location and extent of the meniscal tear. Clinical evaluation remains the basic source of information, with other clinical factors and clinical assessment of the contralateral knee to complete the findings. Complementary investigations such as CT and MRI may also be needed. The ultimate treatment decision should be based on an arthroscopic protocol, which specifies the extent of the lesion and the condition of the other intra-articular structures.

\section{Subdivision of tears based on treatment}

Masterly neglect. Some meniscal tears should be left untreated if they are minimal, not exceeding $1.5 \mathrm{~mm}$ in length, and do not correlate with clinical findings. Small partial-thickness split tears can also be left untreated especially if the patient has a low level of physical activity (Fig. 8).

Suture or excision? This dilemma arises when tears are considered along with the history and the clinical findings. These factors are important in the choice between partial meniscectomy, meniscal suture, meniscal welding (Jackson 1996, personal communication), or partial meniscal replacement (Steadman 1996, personal communication). Only the peripheral $25 \%$ of the meniscus is vascularised, the remainder deriving nutrition from synovial fluid. A tear involving the vascularised area at the junction of the capsule and meniscus is designated a 'red-on-red' tear, one in

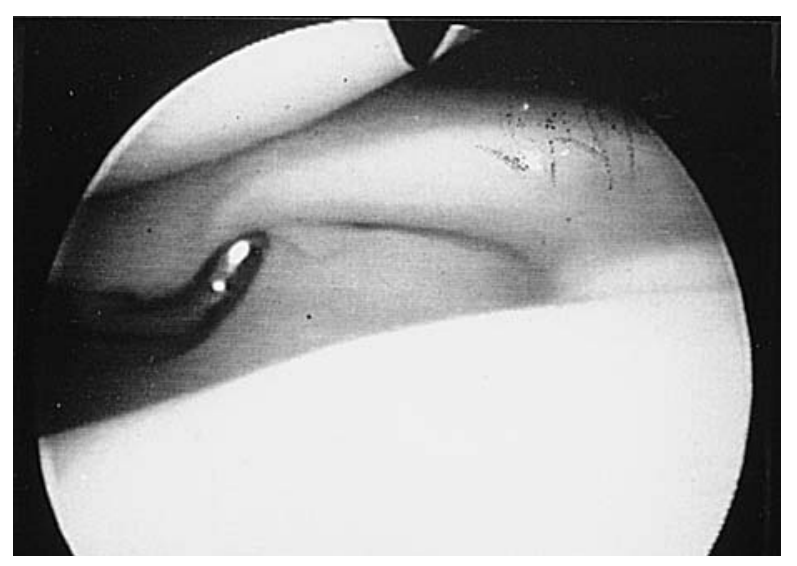

Fig. 8

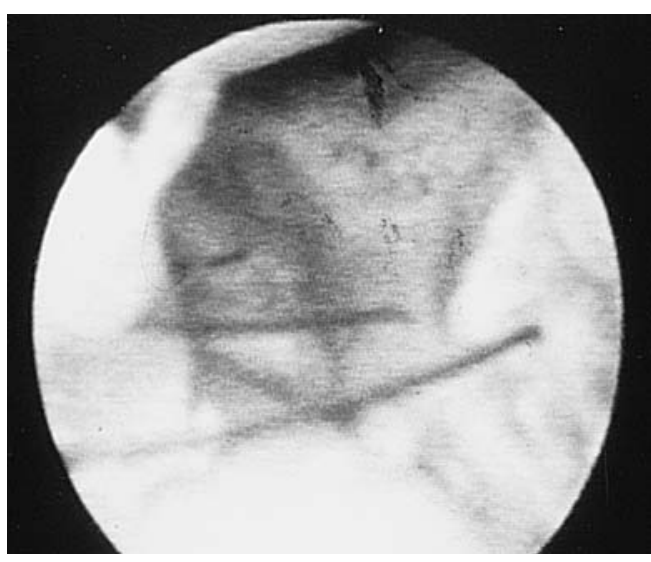

Fig. 9

Figure 8 - A small partial-thickness split tear of the lateral meniscal body can be left untreated, especially if the patient has a low level of physical activity. Figure $9-$ A red-on-red tear mainly involving the medial meniscal rim should always be sutured. 
the avascular, most central, part of the meniscus a 'whiteon-white' tear, and one at the junction between the vascular and avascular areas a 'red-on-white' tear. From my own series, I conclude that red-on-red tears should always be sutured (Fig. 9); they carry the best prognosis because of the vascularity of the meniscus in that area. By contrast, white-on-white tears form an absolute contraindication. Decisive factors in red-on-white tears are the extent, the location and particularly the aspect of the tear, and its acute or chronic nature. Acute tears have a much better prognosis for healing after suture.

\section{ALTERNATIVE TREATMENTS}

Meniscal welding. Laser beams have been used to resect menisci, smooth out articular cartilage, release soft tissue, shrink collagen in shoulder surgery, and remove degenerative discs in spinal surgery. In the future, tissue welding with covalent bonding of collagen fibres by laser-activated photodynamic methods is a distinct possibility. The stimulatory, genetic and mutagenic effects of laser therapy, however, must be studied in greater depth.

Partial meniscal replacement. ReGen Biologics (Sulzer Orthopädie AG, Baar, Switzerland) have developed a collagen-based device designed to stimulate regeneration of meniscal tissue to replace damaged or deficient host tissue. This collagen meniscus implant (CMI) appears to support regeneration of meniscal tissue. In accordance with a concept of guided tissue regeneration the collagen matrix provides a template for cellular infiltration and deposition of a new matrix. If successful, the regenerated tissue would provide many of the functions of the normal meniscus and also protect the joint.

Animal studies in vivo have shown that a CMI can support meniscal regeneration in animal knees, and good or excellent results have been obtained in pigs. Similar results were achieved in $62 \%$ of knees in the first canine study. During this experimental study, improvements were made in implant design and construction, and the cross-linking agent was changed to give more uniformity and stability. Gamma irradiation was used to sterilise the implant thus avoiding the potential toxic residuals from the ethylene oxide sterilisation used previously.

In a clinical feasibility study of the first ten patients the implant supported cellular infiltration and tissue ingrowth. The regenerated tissue at six months had a chondroid-like histological appearance.

\section{MENISCAL TRANSPLANTATION}

In view of the satisfactory clinical results and the physical integrity of the semilunar cartilage obtained in the long term with arthroscopic meniscal suturing techniques, we are considering a treatment for chronic forms of degenerative meniscal pathology. In young patients the deleterious effects of meniscectomy on the load-bearing cartilage are important, as well as the problems after meniscectomy associated with ligamentous rupture in ACL-deficient knees. The treatment of ligamentous instability has been improved by the use of tendon allografts instead of synthetic devices, ${ }^{10}$ and the promising short-term results made us reconsider meniscal transplantation. Meniscal allografts could replace the shock-absorbing and spacer functions which are defective in ACL-deficient knees. Previous attempts using meniscal allografts in dogs gave satisfactory results regarding the fixation and the recovery of normal metabolic and mechanical properties at six months, ${ }^{11,12}$ and their clinical use has been advocated. ${ }^{13,14}$

Meniscal preservation techniques. Various methods can be used to preserve fresh meniscal allografts. Differing results have been obtained with cryopreservation. Shortterm storage does not appear to affect the morphological appearance or biochemical characteristics of the menisci. ${ }^{15}$ Biosynthetic activities, however, are decreased to less than $50 \%$ of normal control values and only $10 \%$ of transplanted meniscal cells have metabolic activity.

The early results obtained with gamma-sterilised, lyophilised meniscal allografts indicated excellent fixation of the meniscal body, but the fine architecture of the meniscus was totally disrupted and the tissue was non-viable. ${ }^{16}$ In solvent-dried menisci the collagen bundle has a fairly normal structure, but they are also non-viable. Because of the successes reported by Zukor et $\mathrm{al}^{14}$ in the transplantation of fresh allografts, we have opted for a fourth option, meniscal culture.

Meniscal culture. Donor menisci are removed in an operating room under strict aseptic conditions during the procurement of other organs from heart-beating (multipleorgan donors) or non-heart-beating donors (Fig. 10). The cold ischaemia time must not exceed 12 hours, during which the meniscus remains viable. Through a transverse arthrotomy, the lateral collateral ligaments and cruciate ligaments are divided and the knee is dislocated anteriorly.

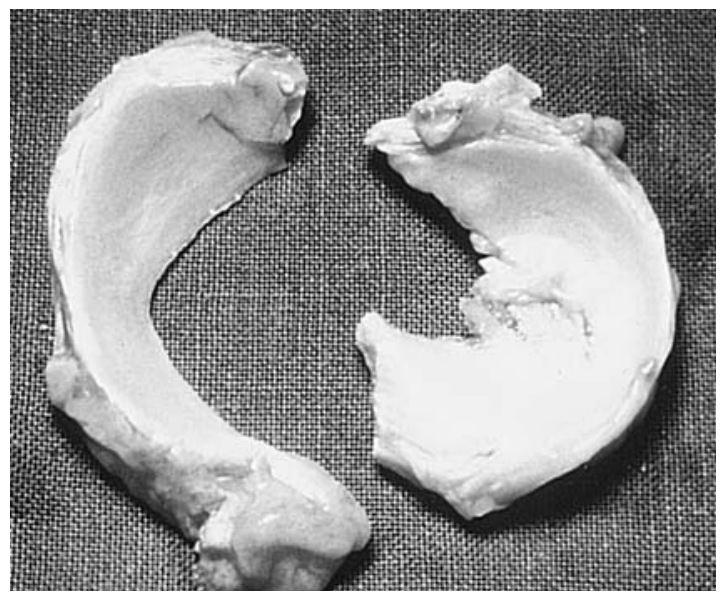

Fig. 10

The medial and lateral menisci are removed with some synovial attachment allowing for atraumatic manipulation. 


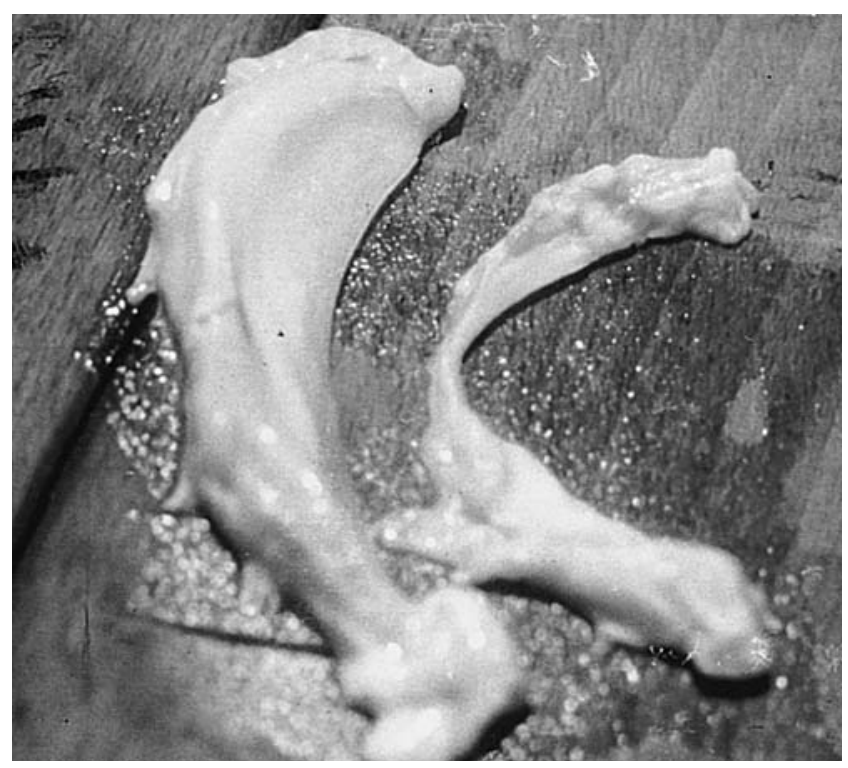

Fig. 11

The original meniscal remnant (right) is removed from the medial compartment. The viable meniscal allograft (left) has a much larger loadbearing surface, which reduces the risk of degenerative cartilaginous changes.

The lateral and medial semilunar cartilages are inspected for macroscopic tears or degenerative changes, and macroscopically intact specimens are removed for experimental investigation or clinical use. Both menisci of each knee are removed with a small synovial rim for manipulation. The meniscus itself is treated in a strictly atraumatic fashion. We have evaluated 23 menisci removed for experimental investigation.

For in vitro culture immediately after harvesting the menisci are placed in Dulbecco's modified Eagle's medium with $0.002 \mathrm{M}$ L-glutamine, $1 / 1000$ antibiotic-antimycotic suspension (streptomycin $10 \mu \mathrm{g} / \mathrm{ml}$, penicillin $10 \mathrm{U} / \mathrm{ml}$, fungizone $0.025 \mu \mathrm{g} / \mathrm{ml}$ ) and $20 \%$ fetal calf serum. The recipient's serum is used for clinical applications. The menisci are stored in a plastic container (DANCON; Teknunc-4000 Roskilde, Denmark) to which $70 \mathrm{ml}$ of incubation medium are added. The containers are placed in a modular incubation chamber (Flow Laboratories, Del Mar, California) at a constant temperature of $37^{\circ} \mathrm{C}$ and under continuous air flow (95\% air and 5\% $\mathrm{CO}_{2}$ ). Humidity is controlled by placing an open receptacle filled with sterile water in the incubation chamber. The incubation media are replaced every three days. ${ }^{17}$

\section{Clinical experience}

Material and methods. Since January 1989 we have treated 60 patients with viable meniscal allografts. The first 40 cases are included in this study terminating in September 1994. The mean follow-up was 3 years 1 month (1 year to 6 years 2 months). There were 31 men and 5 women; 23 had transplantation of the medial meniscus and 17 of the lateral meniscus. The mean age at the time of surgery was 35 years 10 months (23 years 5 months to 49 years 10

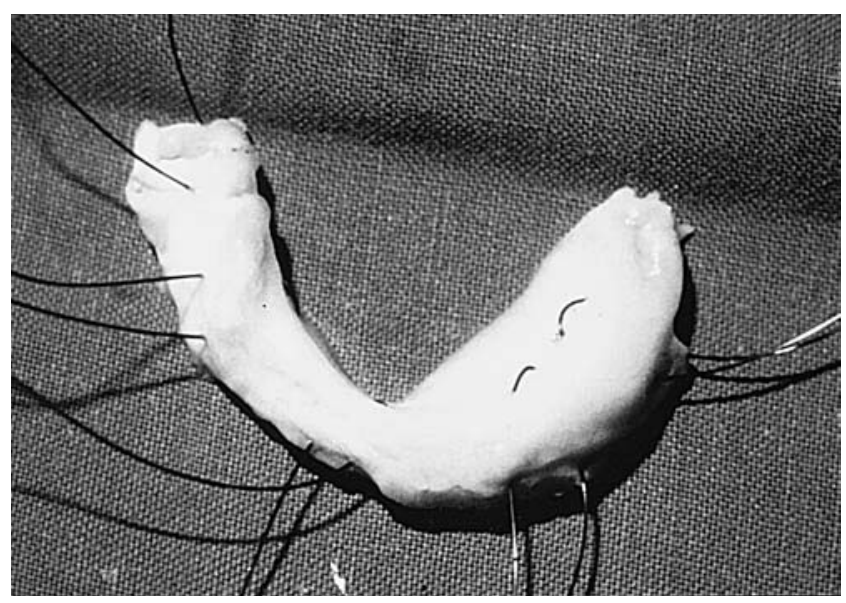

Fig. 12

The meniscal allograft, ready for implantation, is threaded with $6 \times 2.0$ PDS sutures mounted on dual needles (PDS: polydioxanone sutures).

months). Meniscal transplantation was performed on its own in 24 patients, combined with a valgus osteotomy in ten, and with an intra-articular reconstruction of the anterior cruciate ligament using a tendon allograft in one patient. ${ }^{10}$ Lateral meniscal transplantation was associated with a supracondylar femoral varus osteotomy in one patient.

Operative technique. Under epidural anaesthesia the patient is placed supine and a tourniquet applied. Medial anterior arthrotomy is performed and the cartilage of the medial compartment is inspected. In most cases the arthroscopically diagnosed osteoarthrotic changes are confirmed. The small meniscal rim does not provide adequate protection of the load-bearing cartilage and is resected down to the meniscosynovial junction until a potentially bleeding surface of synovial lining is exposed (Fig. 11). Resection of the posterior attachment of the meniscal remnants is difficult through an anterior approach alone, and a posteromedial incision is added to facilitate transplantation and remove the meniscal rim to the posterior aspect of the meniscosynovial junction. These two incisions do not compromise the medial collateral ligament and the whole of the medial compartment can be inspected.

The meniscal allograft is prepared, rinsed and threaded with 5 or $6 \times 2.0$ polydioxanone (PDS) sutures mounted on dual needles (Ethicon; Ethnor J.J, Neuilly, France) (Fig. 12). The allograft is implanted from posterior to anterior. The posterior horn is sutured in position by trans-synovial stitches and the allograft secured to the freshened meniscosynovial lining. Three PDS sutures are usually sufficient to stabilise firmly the posterior portion of the meniscus. At the medial aspect the exposure is extended subcutaneously so that the allograft can be implanted by the same technique. The anterior horn of the medial meniscus is sutured in its original position by two PDS stitches.

The synovium is then closed posteriorly and anteriorly 


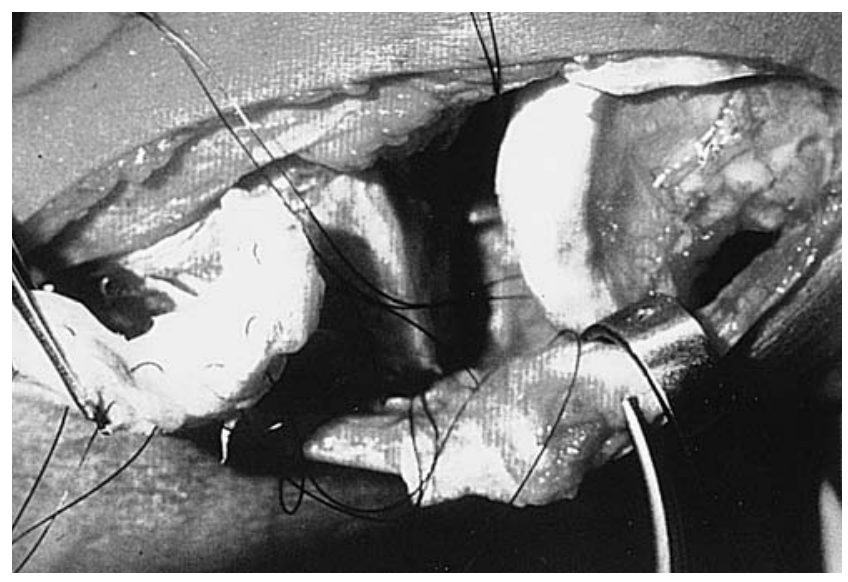

Fig. 13

The threaded viable meniscal allograft is inserted from posterior to anterior. An osteotomy of the femoral insertion of the lateral collateral ligament allows easier access to the posterolateral corner of the lateral side of the knee.

by synoviomeniscal sutures, which provide further stabilisation of the allograft, and a probe is used to confirm that the transplant is in the correct position. The wounds are closed, with drainage, and a plaster cast is applied.

For transplantation of the lateral meniscus a lateral parapatellar arthrotomy is used and the articular cartilage is inspected. The residual rim of the meniscus is resected to obtain a potentially bleeding surface and a second counter incision may be required in the posterolateral corner. The posterior remnants are resected and the threaded allograft is inserted from posterior to anterior and sutured as described for the medial meniscus. The presence of the common peroneal nerve in the posterolateral corner of the knee poses a technical difficulty. Since the popliteus muscle and the lateral collateral ligament are also in this corner, osteotomy and removal of the proximal insertion of the lateral collateral ligament are an efficient alternative (Fig. 13). This facilitates removal of the meniscal remnants and implantation of the lateral meniscal allograft without jeopardising the common peroneal nerve.

In the first ten cases we used immobilisation in a plaster cast for six weeks, but this period was progressively reduced. Rehabilitation is now started immediately after surgery; we use plaster-cast immobilisation only in those patients who are not willing to comply with the treatment protocol or for eight days for pain relief. Flexion-extension exercises without rotatory load are encouraged. After six weeks progressive weight-bearing is allowed.

Clinical evaluation. We attempted to use an objective rating system to evaluate the results of meniscal allografting. The operative technique of meniscal transplantation is itself straightforward, but in 12 of our 40 cases additional procedures were performed, consisting of an osteotomy in 11 and an ACL-plasty in one. These combined interventions cannot be assessed using ligamentoplasty rating systems. ${ }^{18,19}$

We therefore considered that meniscal transplantation,

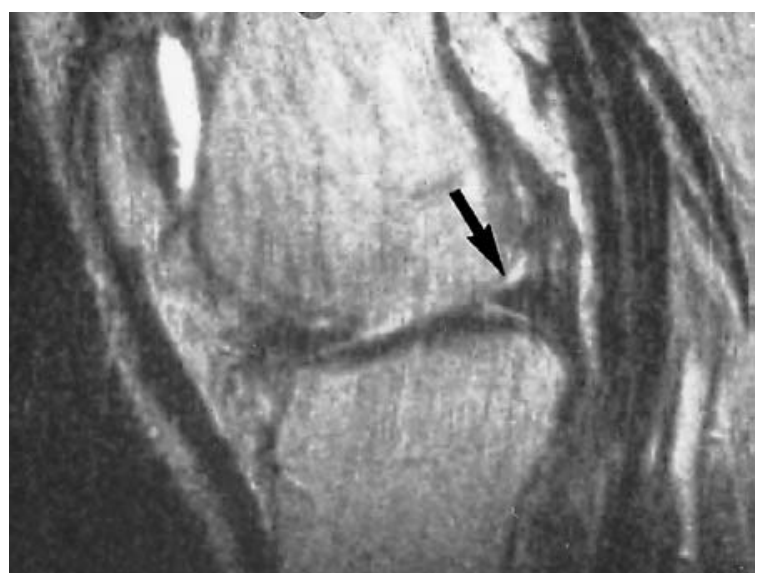

Fig. 14

Homogeneous signal intensity on MRI six months after medial meniscal transplantation. A small intra-articular effusion is present.

performed as an isolated or combined procedure, was comparable with an arthroplasty, and used the Hospital for Special Surgery (HSS) knee rating system ${ }^{20}$ to assess pain, stability and function. Flexion and extension contractures, malalignment and the use of crutches scored minus points.

This evaluation was correlated with the objective findings of imaging. MRI of the menisci was essential to try to confirm ingrowth of the allografted material to the synovial wall. MRI also provides information on cartilage quality and ligament reconstruction. All 40 patients in our study had MRI, although not at regular intervals (Fig. 14). Arthroscopic evaluation was performed only to obtain material for histological studies and to assess the aspect and firmness of meniscal fixation.

Complications. Four patients had transient synovitis; white cell counts suggested mechanical synovitis in three and inflammatory synovitis in one. The mechanical synovitis resolved spontaneously after a short period of time. The patient with inflammatory synovitis had an abnormal white cell count, but eventually regained normal function of the knee. Other signs of infection also returned to normal levels. Manipulation under anaesthesia was necessary in three patients.

Results and discussion. There were no strict selection criteria for including patients in our study. Young patients with mechanical complaints who had already undergone one or several meniscectomies were considered for inclusion. Only one patient complained of knee instability due to ACL deficiency. In 11 patients, mechanical complaints were ascribed to manifest axial malalignment. Because the patient population was too divergent we did not regularly use scoring systems in the evaluation of the 40 cases.

The principal indication was intractable pain not responding to analgesics. Based on the experiments of Wirth et $\mathrm{al}^{13}$ and Zukor et $\mathrm{al}^{14}$ we offered them meniscal 


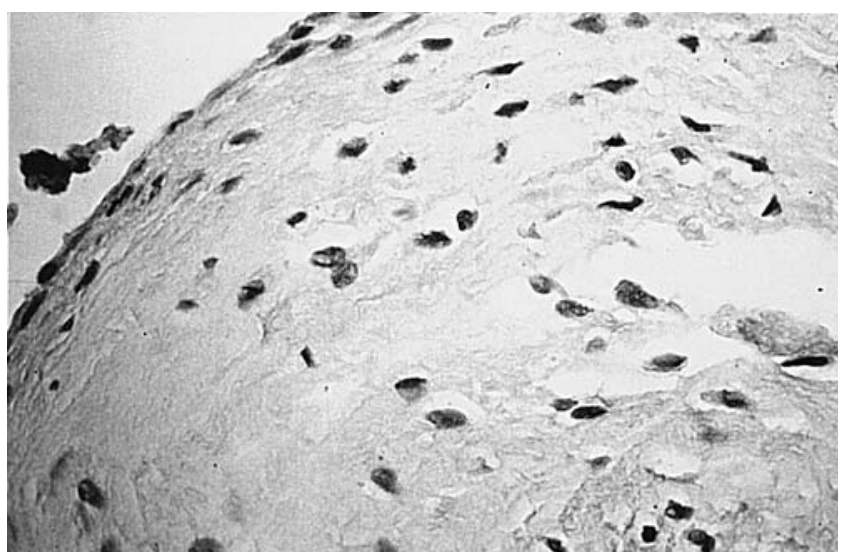

Fig. 15

Viable cells are present in the anterior horn four months postoperatively. Some fibrin covering is observed (haematoxylin and eosin x 220).

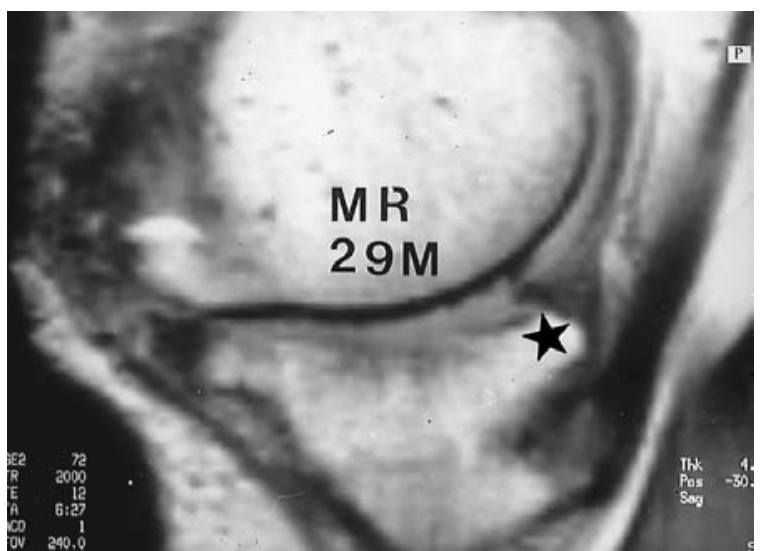

Fig. 16

MRI showing that the medial meniscal transplant is firmly fixed to the meniscosynovial junction in the posteromedial corner*. Slight atrophy of the medial meniscal anterior horn is also shown.

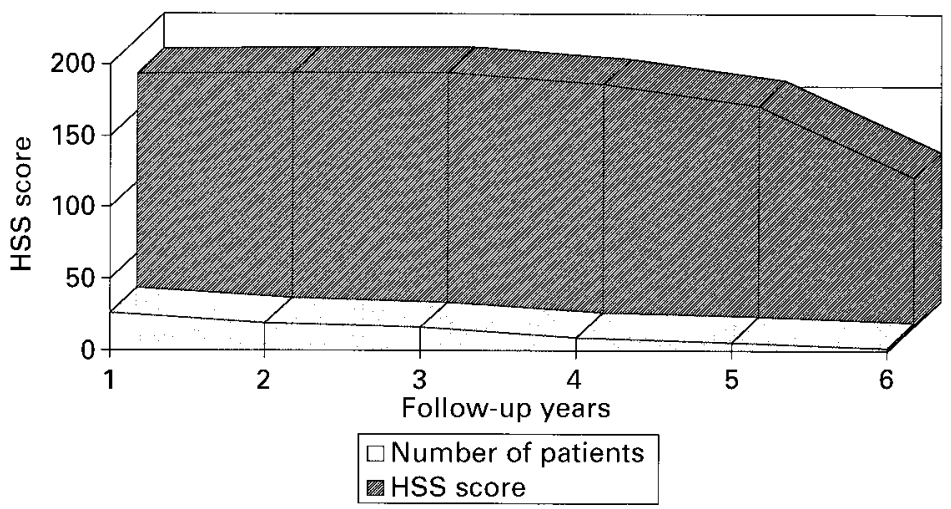

Fig. 17

The mean HSS score decreased slowly and slightly, especially between the fifth and sixth years.

transplantation.

In deep-frozen or gamma-sterilised, lyophilised menisci, there are some alterations to the structural integrity of the meniscal body. Because of the potential for disease transmission implantation of allografts must not be delayed for too long after harvesting. The work of Verbruggen ${ }^{17}$ and Verbruggen et $\mathrm{al}^{21}$ on cartilage metabolism and cartilage cell structures allowed us to culture the entire harvested semilunar cartilages so that viable material could be implanted.

It has been established that fibrochondrocytes in a collagen structure migrate from the synovial rim $^{22}$ and we therefore assumed that the production of proteoglycan would be more efficient if viable material could be implanted on the same day as harvest. Proteoglycan production was studied in vitro in meniscal cultures in medium supplemented with FCS. It was found to be maximal, both qualitatively and quantitatively, after a culture period of 10 to 14 days.

No major clinical complications were encountered in our series of 40 patients, although not all had the same operative procedures. Repeat arthroscopy was performed on 12 patients at up to two years after surgery; all showed viable meniscal tissue, both macroscopically and microscopically (Fig. 15).

DNA fingerprinting of the cells cultured from the harvested material in the same 12 patients showed matching in four patients, non-matching in three and both matching and non-matching in one. This means that at a two-year follow-up slow ingrowth had occurred in the meniscal transplant while some donor cells were still surviving, suggesting chemical activity in the meniscal transplant from the first day of transplantation. These findings were confirmed on MRI; no major differences in contrast were seen (Fig. 16).

The mean HSS score decreased slowly and slightly, especially between the fifth and sixth years (Fig. 17). In the first year, an arbitrarily chosen score of over 175 points was achieved in $72 \%$ of the patients. After four years, $67 \%$ of the patients still scored higher than 175 , $22 \%$ scored between 100 and 175, and $11 \%$ scored below 100. Three patients who had received five meniscal transplants (two bilateral) eventually had total knee arthroplasty. 
Table I. Statistical results for function in viable meniscal transplants

\begin{tabular}{ll}
\hline & p value* \\
\hline Pain & 0.0000 \\
Stability & \\
$\quad$ Anteroposterior & 0.0099 \\
$\quad$ Mediolateral & 0.412 \\
Gait & 0.0066 \\
Stair-climbing & 0.0034 \\
ROM & 0.2188 \\
Flexion contracture & 0.1936 \\
Extension lag & 0.2568 \\
\hline
\end{tabular}

$*<0.05$ is significant

Statistical analysis using the Wilcoxon matched-pair signed-rank test showed significant differences, with marked improvement in pain, postoperative antero-

\section{REFERENCES}

1. Jackson RW. The role of arthroscopy in the management of the arthritic knee. Clin Orthop 1974;101:28-35.

2. De Meulemeester C, Verdonk R, Van Eetvelde G, Meire D. The value of CT scan in the evaluation of meniscal sutures. Proceedings of the 103rd Annual Meeting of the American Orthopaedic Association, Boston, Massachussetts, 1990, 103.

3. Fairbank TJ. Knee joint changes after meniscectomy. J Bone Joint Surg [Br] 1948;30-B:664-70.

4. Lemaire R. Osteoarthritis of the knee joint: a predictable consequence of meniscectomy in patients with angular deformity of the knee. Acta Chir Belg 1977;76:355-60.

5. Kettelkamp DB, Jacobs AW. Tibiofemoral contact area: determination and implications. J Bone Joint Surg [Am] 1972;54-A:349-56.

6. Ahmed AM, Burke DL. In vitro measurement of static pressure distribution in synovial joints. Part I: tibial surface of the knee. $J$ Biomech Eng 1983;105:216-25.

7. DeHaven KE. The role of the meniscus. In: Ewing JW, ed. Articular cartilage and knee joint function: basic science and arthroscopy. New York: Raven Press, 1990:103-15.

8. Levy IM, Torzilli PA, Warren RF. The effect of medial meniscectomy on anterior-posterior motion of the knee. J Bone Joint Surg [Am] 1982; 64-A:883-8.

9. Dejour H, Bonnin M, Neyret P. Anterior cruciate deficient knee stability in monopodal stance: the influence of the posterior slope of the tibial plateau of the medial meniscus and of the posteromedial corner. Procs 4th Congress of the European Society of Knee Surgery and Arthroscopy, Stockholm, 1990:44-5.

10. Arnauw G, Verdonk R, Harth A, et al. Prosthetic versus tendon allograft replacement of ACL-deficient knees. Acta Orthop Belg 1991;57,Suppl II:67-74.

11. Arnoczky SP, Cuzzell JZ, McDevitt CA, Torzilli PA, Warren RF, Kristincz TI. Meniscal replacement using a cryopreserved allograft: an experimental study in the dog. Trans Orthop Res Soc 1984; 9:220. posterior and mediolateral stability, gait and stair-climbing (Table I). There was no difference in the pre- and postoperative range of motion, flexion contracture or extension lag (Table I).

We do not yet know whether the long-term survival of donor cells, with the slow ingrowth of host cells into the transplanted meniscus, will have any effect on the longterm functional results. Postoperative follow-up is much too short to allow valid conclusions. From a technical viewpoint, however, allografting appears to have no negative effect on the clinical findings.

The author acknowledges and appreciates the contributions of Erwin Groessens, MD and Tom Lootens, MD. He wishes to thank Iris Wojtowicz and Monique De Pauw for the research and typing of this text, and Marc De Ganck for the figures. He also wishes to thank Robert W. Jackson, MD for permission to include his research on meniscal welding, and Richard Steadman, MD for permission to include his work on partial meniscal replacement.
12. Weismeier K, Wirth CJ, Milachowski KA. Transplantation of the meniscus: experimental study. Rev Chir Orthop 1988;74:155-9.

13. Wirth CJ, Milachowski KA, Weismeier K. Meniscus transplantation in animal experiments and initial clinical results. Z Orthop 1986; 124:508-12.

14. Zukor DJ, Cameron JC, Brooks PJ, et al. The fate of human meniscal allografts. In: Ewing JW, ed. Articular cartilage and knee joint function. New York, etc: Raven Press, 1990:147-52.

15. Arnoczky SP, McDevitt CA, Schmidt MB, Mow VC, Warren RF. The effect of cryopreservation on canine menisci: a biochemical, morphologic and biomechanical evaluation. J Orthop Res 1988; $6: 1-12$.

16. Schmid A, Schmid F, Tiling T. Electron microscopic studies on human meniscal tissue preserved for transplantation. In: Müller W, Hackenbruch W, eds. Proceedings of the second European Congress of Knee Surgery and Arthroscopy. Heidelberg:Springer-Verlag, 1988: 703-7.

17. Verbruggen G. Reparatieprocessen en katabole fenomenen in humaan articulair kraakbeen. Proefschrift tot het verkrijgen van de graad van geaggregeerde voor het hoger onderwijs, Gent, 1990.

18. Lysholm J, Gillquist $\mathbf{J}$. Evaluation of knee ligament surgery results with special emphasis on use of a scoring scale. Am J Sports Med 1982; 10:150-4.

19. Tegner Y, Lysholm J. Rating systems in the evaluation of knee ligament injuries. Clin Orthop 1985;198:43-9.

20. Insall JN, Dorr LD, Scott RD, Scott WN. Rationale of the Knee Society clinical rating system. Clin Orthop 1989;248:13-4.

21. Verbruggen G, Veys EM, Malfait AM, et al. Proteoglycan metabolism in tissue cultured human articular cartilage: influence of piroxicam. J Rheumatol 1989;16:355-62.

22. Stone KR, Rodkey WG, Webber RJ, McKinney L, Steadman JR. Future directions: collagen-based prostheses for meniscal regeneration. Clin Orthop 1990;252:129-35. 